\title{
Association between serum osteocalcin and glucose/lipid metabolism in Chinese Han and Uygur populations with type 2 diabetes mellitus in Xinjiang: two cross- sectional studies
}

Yuan Chen ${ }^{1,2+}$, Qiang Zhao ${ }^{3,4+}$, Guoli Du ${ }^{5}$ and Yancheng X ( $^{{ }^{*}}$

\begin{abstract}
Background: Recent studies have shown osteocalcin (OC) plays an important role in regulating glucose and lipid metabolism. Thus, the aim of this study was to investigate the association of OC with glucose and lipid metabolism in patients with type 2 diabetes mellitus (T2DM) in the Chinese Han and Uygur population.

Methods: A total of 1397 T2DM patients (705 Han and 692 Uygur T2DM patients) were enrolled in the present study. Lipid profile, glucose metabolic indices and total OC (TOC) were measured. Homeostasis model assessment of $\beta$-cells function (HOMA- $\beta$ ), insulin sensitivity (HOMA-IS) and insulin resistance (HOMA-IR) were also calculated in all participants. Pearson/Spearman correlation analysis and multivariate stepwise regression analysis were adopted to test the relationships between $\mathrm{OC}$ and those parameters.

Results: Uygur T2DM patients had significantly higher body mass index (BMI), hemoglobin A1C (HbA1C) and lower TOC compared with their Han counterparts (all $P<0.05$ ). HbA1C was negatively associated with TOC in all Uygur and Han T2DM patients (Total: Uygur: $t=-3.468, P=0.001$; Han: $t=-4.169, P<0.001$ ). BMI was inversely associated with TOC in all Uygur T2DM patients (Males: $t=-2.893, P=0.014$; Females: $t=-2.250, P=0.027$, respectively). Multivariate stepwise regression analysis showed that TOC was positively correlated with HOMA- $\beta$ in the Uygur male group $(\beta=2.101, P=0.040)$ and negatively associated with BMI in all Uygur T2DM patients (Males: $\beta=-1.563, P=0.011$; Females: $\beta=-1.284, P=0.016$, respectively). No significant differences were observed between TOC and lipid profiles in all participants (all $P>0.05$ ).

Conclusion: There were differences in the associations between TOC and glucose metabolism in Han and Uygur T2DM patients, indicating genetic factors may play a role in modulating OC and glucose metabolism in different ethnic population.
\end{abstract}

Keywords: Osteocalcin, Glucose/lipid metabolism, Diabetes mellitus, Han, Uygur

\footnotetext{
* Correspondence: xj1100901@whu.edu.cn

${ }^{\dagger}$ Equal contributors

${ }^{1}$ Department of Endocrinology, Zhongnan Hospital of Wuhan University, 169

Donghu Road, Wuhan, Hubei 430071, China

Full list of author information is available at the end of the article
} 


\section{Background}

Diabetes mellitus (DM), caused by the interaction of insulin resistance (IR) and insulin deficiency, is a common chronic disease in adults worldwide. The incidence of type 2 DM (T2DM) has been steadily increasing in China over the past few decades. Diabetic osteopathy is a common complication among diabetic patients, characterized by a higher risk of bone fracture, osteoporosis and increased brittleness of bone [1].

Nowadays, bone has been identified as an endocrine organ which plays an important role in the energy metabolism. As an osteoblast-derived hormone, osteocalcin (OC) is an indicator of both osteoblast activity and bone formation. Both carboxylated and uncarboxylated forms of OC are found in serum, with the undercarboxylated OC (ucOC) being the main component of total OC (TOC). Recently, increasing attention has been directed toward the role of $\mathrm{OC}$ in modulating glucose and lipid metabolism. Lee et al. showed that $\mathrm{OC}$ is a bone messenger affecting both adipocytes and insulin-producing $\beta$-cells. It increases $\beta$-cells proliferation, insulin secretion, insulin sensitivity, adiponectin expression and energy expenditure by upregulating the expression of adiponectin gene in adipocytes [2]. Adiponectin and its receptors are expressed in human osteoblasts. Adiponectin stimulates the proliferation, differentiation and mineralization of osteoblastic cells $[3,4]$. As one of adipocytokines, adiponectin also enhances insulin sensitivity, indicating there is an interaction among bone, adiposederived factors and glucose/lipid metabolism.

Several clinical studies have investigated the association between OC and glucose/lipid metabolism [5-8]. However, the results were inconsistent, probably owing to the different ethnic origin which is specific to the study population enrolled in the different studies. There were few studies investigating the differential patterns for regulating glucose and lipid metabolism by $\mathrm{OC}$ in Chinese Han and Uygur T2DM patients, so we perform this analysis to investigate the association of serum TOC with glucose and lipid metabolism in Chinese Han and Uygur T2DM patients.

\section{Methods}

\section{Ethical approval of the study protocol}

This study protocol was approved by the Ethics Committee of the People's Hospital of Xinjiang Uygur Autonomous Region. All participants provided written informed consent to participate the research.

\section{Study design and population}

Two independent cross-sectional studies conducted at the Xinjiang Uygur Autonomous Region were included. A total of 1397 Han and Uygur T2DM patients admitted to the endocrine department of the People's Hospital of Xinjiang Uygur Autonomous Region between January 2014 and October 2015 were recruited. Individuals were excluded from this study if they have: acute or severe chronic DM complications, current cancer, chronic/ acute kidney or liver disease, bone-altering conditions (hyperparathyroidism, bilateral hip surgery), oral medications known to influence bone and calcium metabolism, such as vitamin D, bisphosphonates and estrogen.

\section{Definition of DM and its risk factors}

DM was defined as history of DM or was taking antidiabetic medications or had fasting/non-fasting glucose $\geq 7.0 \mathrm{mmol} / \mathrm{L} / \geq 11.1 \mathrm{mmol} / \mathrm{L}$. Body mass index (BMI) was calculated by dividing the weight in kilograms by the height in meters squared. Waist circumference (WC) was measured at midpoint between the last palpable rib and the suprailiac crest using a measuring tape parallel to the floor with the subjects standing and breathing normally. Obesity was defined as BMI $\geq 28.0 \mathrm{~kg} / \mathrm{m}^{2}$ [9].

\section{Anthropometric measurements}

Fasting blood samples were collected from all participants for the assessment of biochemical indices. Fasting plasma glucose (FPG), glycated hemoglobin A1C (HbA1C), total cholesterol (TC), low density lipoprotein-cholesterol (LDL-C), high density lipoprotein-cholesterol (HDL-C), triglycerides (TG), calcium, phosphorus, alkaline phosphatase (ALP), parathyroid hormone (PTH), 25-hydroxyvitamin D3 [25(OH)VD3], procollagen type I N-terminal propeptide (P1NP) and $\beta$-C-terminal telopeptide of type I collagen $(\beta-\mathrm{CTX})$ were measured using standard enzymatic methods in the central laboratory of the People's Hospital of Xinjiang Uygur Autonomous Region. Fasting plasma insulin (FINS)/C-peptide (FCP), 2 h-post OGTT plasma glucose $(2 \mathrm{~h}-\mathrm{PG})$ and $\mathrm{C}$-peptide $(2 \mathrm{~h}-\mathrm{CP})$ were measured using enzyme-linked immunosorbent assay method. The assessment of insulin sensitivity was estimated by homeostasis model assessment-IR (HOMA-IR) based on FPG and insulin measurements as follows: HOMA-IR $=[$ FINS $(\mathrm{mU} / \mathrm{L}) \times$ FPG $(\mathrm{mmol} / \mathrm{L})] / 22.5$ [6] Homoeostasis model assessment for insulin secretion (HOMA-IS) was calculated using the formulas previously described [10]. To estimate $\beta$-cells function, HOMA- $\beta$ was calculated as follows: $(20 \times$ FINS)/(FPG-3.5). Serum TOC was measured using the electrochemical luminescence method. Bone mass density (BMD) was measured using dual-energy x-ray absorptiometry (LINK Osteocore 3, France) with intra- and inter-assay coefficient of variation below 1\%. BMD at the hip, anteroposterior lumbar spine (L1-4), greater trochanter (GT) and femoral neck were measured (grams per square centimeter).

\section{Statistical methods}

All statistical analyses were performed with the SPSS for Windows (version 17.0, SPSS Inc., Chicago, IL, USA). 
Continuous data were examined for normality and expressed as mean \pm standard deviation (SD) or median and compared with the Student's t test or Wilcoxon test as appropriate. Categorical variables were summarized as percentages and compared with the Chi-square test. Pearson/Spearman analysis model was used to assess the association of TOC with anthropometric variables, glucose and lipid metabolic indices. A multivariate stepwise analysis was performed to test for the association between TOC and other independent variables after adjustment for confounding variables. A 2 -sided $P<0.05$ was considered to indicate statistical significance.

\section{Results}

During the study period, a total of 705 Han T2DM patients (59.12 \pm 8.77 years, $47.98 \%$ men) and 692 Uygur T2DM patients (59.95 \pm 7.38 years, $49.50 \%$ men) were enrolled in the present study.

\section{Baseline characteristics of the study population stratified by ethnicity and sex}

Clinical characteristics of all participants stratified by ethnicity and sex were summarized in Table 1. There were no differences between Han and Uygur T2DM patients with respect to age, course of disease, systolic blood pressure (SBP), diastolic blood pressure (DBP), BMD-neck, BMD-GT, BMD (L1-4), previous medications and prevalence of comorbidities. For total, males and females, Uygur T2DM patients had higher WC, BMI and higher prevalence of obesity compared with their Han counterparts (all $P<0.05$ ). In the female group, Uygur females had significantly higher BMDneck values than Han females $\left(0.73 \pm 0.11 \mathrm{~g} / \mathrm{cm}^{2}\right.$ vs. $\left.0.70 \pm 0.14 \mathrm{~g} / \mathrm{cm}^{2}, P=0.024\right)$.

\section{Laboratory characteristics of the study population}

Table 2 showed the laboratory findings of the study stratified by ethnicity and sex. There were no differences with respect to the PTH, calcium, FCP, FINS, HDL-C, PINP, HOMA-IR, HOMA-IS and HOMA- $\beta$ between Han and Uygur T2DM patients (all $P>0.05$ ). Han T2DM patients had significantly higher TOC compared with Uygur participants (Total: $19.30 \pm 12.53 \mathrm{ng} / \mathrm{mL}$ vs. $13.91 \pm 7.45 \mathrm{ng} / \mathrm{mL}, P<0.001$; Males: $19.10 \pm 11.71 \mathrm{ng} /$ $\mathrm{mL}$ vs. $12.69 \pm 7.22 \mathrm{ng} / \mathrm{mL}, \quad P<0.001$; Females: $19.60 \pm 12.33 \mathrm{ng} / \mathrm{mL}$ vs. $15.42 \pm 7.46 \mathrm{ng} / \mathrm{mL}, P<0.001)$. For total participants, males and females, Uygur T2DM patients had significantly higher FPG, 2 h-PG, high sensitivity C-reactive protein (hs-CRP) and HbA1C than their Han counterparts (all $P<0.05$ ). In the total and female groups, Uygur T2DM patients had higher ALP, LDL-C, TC, TG and $\beta$-CTX and lower 25(OH)VD3, creatinine and $2 \mathrm{~h}-\mathrm{CP}$ (all $P<0.05)$.

\section{Pearson/Spearman correlation analysis}

The correlations between TOC and anthropometric variables, glucose and lipid metabolic parameters were presented in Tables 3. BMI was inversely correlated with TOC in all Uygur T2DM patients (Total: $r=-0.118, P=0.003$; Males: $r=-0.168, P=0.025$; Females: $r=-0.164$, $P=0.030)$, while it was only negatively associated with TOC in the Han total and Han female group (Total: $r=-0.119, P=0.004$; Females: $r=-0.262, P<0.001)$. WC was demonstrated to be inversely associated with TOC only in the Han female group $(r=-0.181, P=0.004)$. HbA1C was negatively associated with TOC in all T2DM patients (Total Uygur: $r=-0.143, P<0.001$; total Han: $r=-0.181$, $P<0.001$; Uygur males: $r=-0.171, P=0.002$; Han males: $r=-0.149, P=0.008$; Uygur females: $r=-0.215, P<0.001$; Han female: $r=-0.207, P<0.001) .2$ h-PG was inversely related with TOC in all Han and Uygur female participants, while FPG was inversely associated with TOC in the Han and Uygur female group (all $P<0.05$ ). When it comes to P1NP, PTH and phosphorus, they were positively associated with TOC in all Han and Uygur T2DM patients (all $P<0.05)$. There was no significant correlation between serum TOC and lipid metabolic parameters, including TC, LDL-C and TG in all Han and Uygur T2DM patients.

\section{Multivariate stepwise regression analysis}

Multivariate stepwise regression analysis of the associations between TOC and multiple parameters stratified by ethnicity and sex were presented in Table 4 . In a multivariate stepwise regression model with TOC as the dependent variable, we identified $\mathrm{HbA1C}$ was negatively correlated with TOC in all Han and Uygur participants. BMI was inversely associated with TOC in all Uygur participants, including males and females. While HOMA- $\beta$ was only positively associated with TOC in the Uygur male group. In addition, $\beta$-CTX was positively associated with TOC in all Han and Uygur participants.

To investigate the association between independent risk factors of $\mathrm{HbA1C}$, BMI and HOMA- $\beta$, a multivariate regression analysis was performed with $\mathrm{HbA1C}$ as the dependent variable and age, BMI, TOC, FPG, FINS, FCP, TG, TC, HDL-C and LDL-C as independent variables. We identified a significantly negative association between TOC and HbA1C in all participants (Han: $\beta=-0.045, P=0.015$; Uygur: $\beta=-0.034, P=0.014$ ) (Table 5). While in a multivariate stepwise regression analysis performed with BMI as the dependent variable, we found TOC was negatively associated with BMI only in the Uygur groups (Males: $\beta=-1.563, P=0.011$; Females: $\beta=-1.284, P=0.016$ ) (Table 6). Furthermore, TOC was positively associated with HOMA- $\beta$ in the Uygur male group after adjusting for confounding variants $(\beta=2.101, P=0.040)$ (Table 6). 


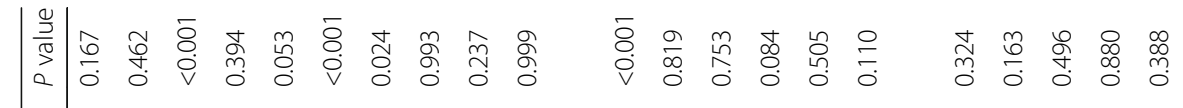

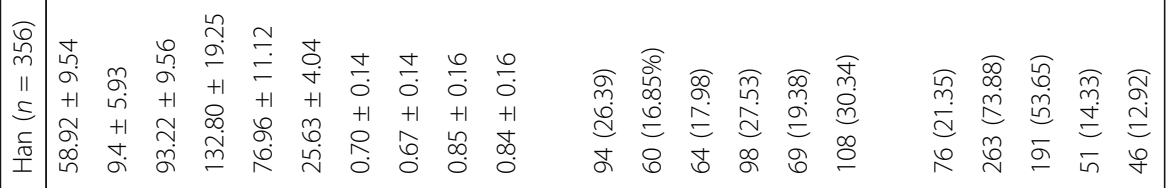

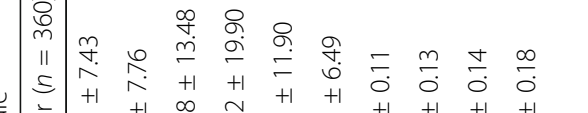

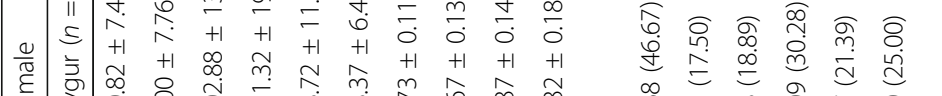

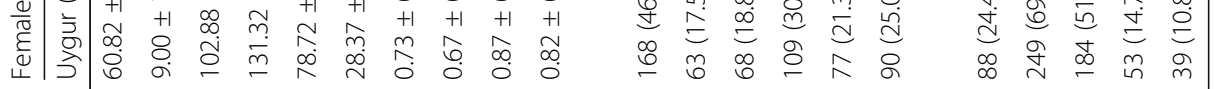

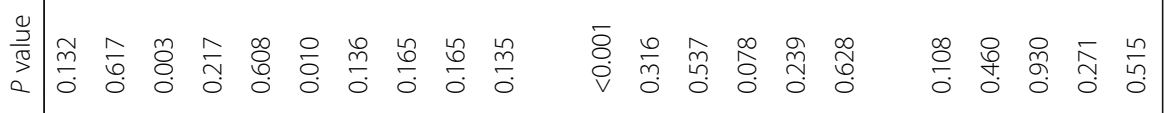
๙ิ

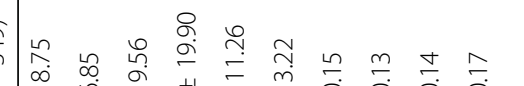

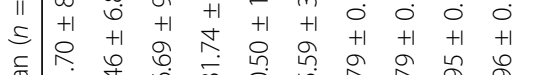

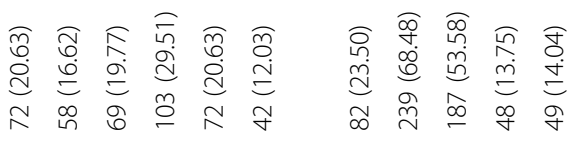

$\underset{\widetilde{N}}{m}$

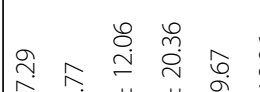

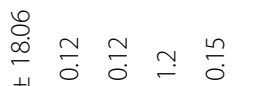

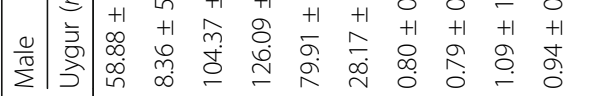

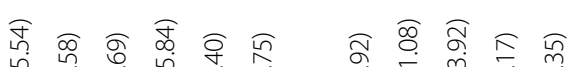

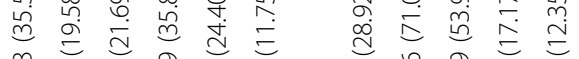

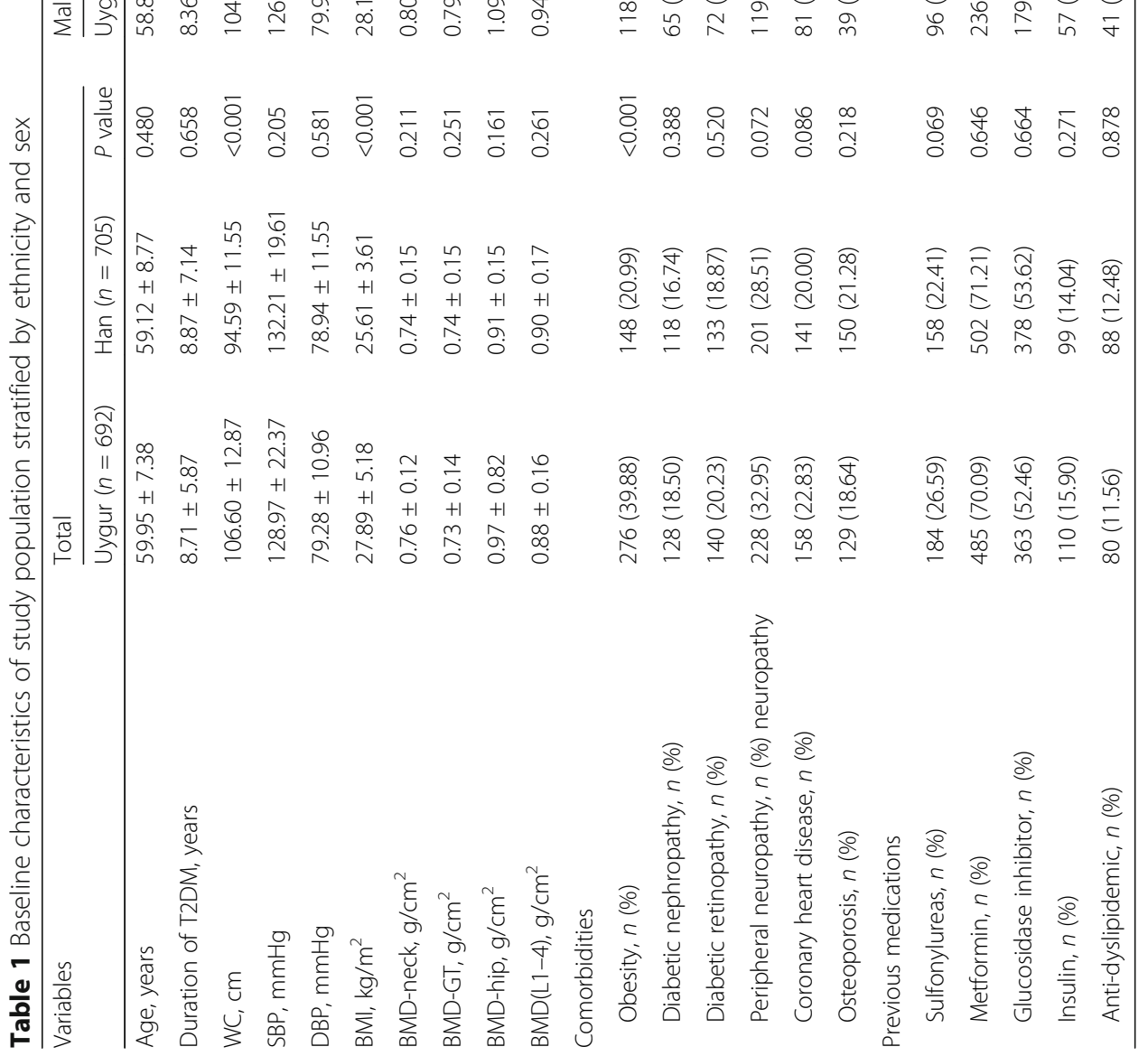




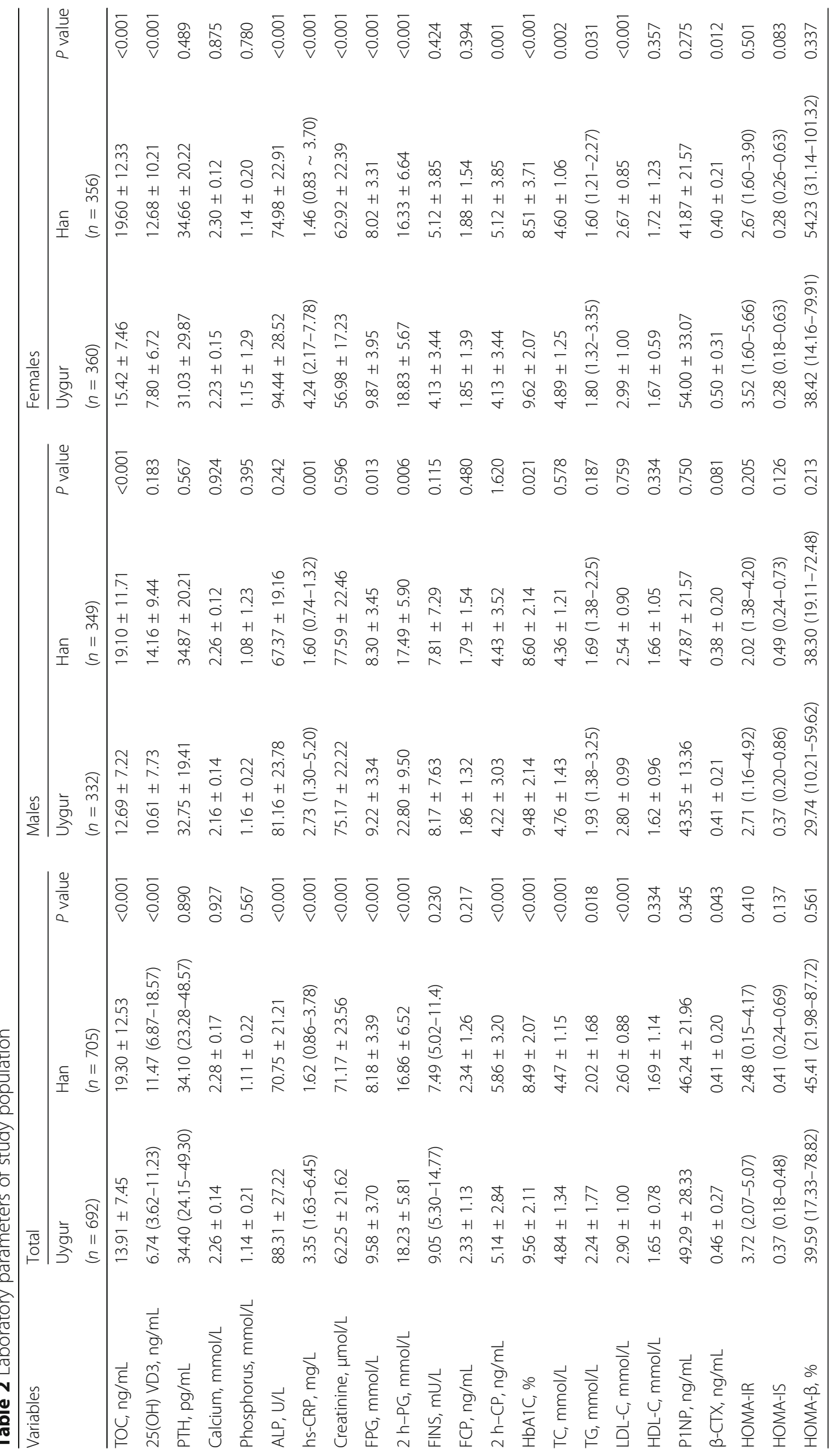


Table 3 Spearman/Pearson analysis between TOC and glucose/lipid metabolic indices

\begin{tabular}{|c|c|c|c|c|c|c|c|c|c|c|c|c|}
\hline \multirow[t]{3}{*}{ Variables } & \multicolumn{4}{|l|}{ Total } & \multicolumn{4}{|l|}{ Males } & \multicolumn{4}{|l|}{ Females } \\
\hline & \multicolumn{2}{|l|}{ Uygur } & \multicolumn{2}{|l|}{ Han } & \multicolumn{2}{|l|}{ Uygur } & \multicolumn{2}{|l|}{ Han } & \multicolumn{2}{|l|}{ Uygur } & \multicolumn{2}{|l|}{ Han } \\
\hline & $r$ & $P$ & r & $P$ & $r$ & $P$ & $r$ & $P$ & $r$ & $P$ & r & $P$ \\
\hline Age & -0.047 & 0.259 & -0.024 & 0.537 & 0.200 & 0.733 & 0.210 & 0.712 & 69.000 & 0.184 & 0.110 & 0.860 \\
\hline Duration of T2DM & 0.038 & 0.329 & 0.047 & 0.259 & 0.134 & 0.210 & -0.040 & 0.480 & 0.050 & 0.340 & 0.134 & 0.310 \\
\hline BMI & -0.118 & 0.003 & -0.119 & 0.004 & -0.168 & 0.025 & -0.049 & 0.390 & -0.164 & 0.030 & -0.262 & $<0.001$ \\
\hline WC & -0.026 & 0.514 & -0.128 & 0.002 & -0.039 & 0.503 & -0.012 & 0.829 & -0.080 & 0.132 & -0.181 & 0.004 \\
\hline 25(OH) VD3 & 0.025 & 0.435 & -0.019 & 0.665 & 0.019 & 0.763 & -0.012 & 0.838 & 0.052 & 0.347 & 0.001 & 0.987 \\
\hline PTH & 0.162 & $<0.001$ & 0.220 & $<0.001$ & 0.135 & 0.031 & 0.263 & $<0.001$ & 0.180 & 0.001 & 0.187 & 0.004 \\
\hline Calcium & -0.002 & 0.965 & -0.003 & 0.946 & -0.073 & 0.291 & 0.086 & 0.182 & 0.057 & 0.350 & 0.022 & 0.757 \\
\hline Phosphorus & 0.217 & $<0.001$ & 0.167 & $<0.001$ & 0.204 & 0.003 & 0.500 & 0.444 & 0.228 & $<0.001$ & 0.273 & $<0.001$ \\
\hline ALP & 0.042 & 0.538 & 0.043 & 0.470 & -0.020 & 0.807 & 0.032 & 0.699 & 0.099 & 0.185 & -0.024 & 0.785 \\
\hline FPG & -0.115 & 0.003 & -0.125 & 0.003 & -0.078 & 0.179 & -0.080 & 0.168 & -0.152 & 0.004 & -0.165 & 0.009 \\
\hline $2 \mathrm{~h}-\mathrm{PG}$ & -0.132 & 0.002 & -0.127 & $<0.001$ & -0.105 & 0.092 & -0.178 & 0.030 & -0.166 & 0.003 & -0.159 & 0.016 \\
\hline FINS & 0.064 & 0.254 & 0.084 & 0.077 & 0.002 & 0.983 & 0.111 & 0.185 & 0.096 & $<0.001$ & 0.035 & 0.699 \\
\hline FCP & 0.009 & 0.831 & 0.069 & 0.134 & 0.164 & 0.009 & 0.192 & 0.002 & 0.183 & 0.002 & 0.076 & 0.263 \\
\hline $2 \mathrm{~h}-\mathrm{CP}$ & 0.057 & 0.192 & 0.077 & 0.095 & -0.068 & 0.289 & 0.088 & 0.163 & 0.168 & 0.005 & 0.037 & 0.594 \\
\hline $\mathrm{HbA1C}$ & -0.143 & $<0.001$ & -0.181 & $<0.001$ & -0.171 & 0.002 & -0.149 & 0.008 & -0.215 & $<0.001$ & -0.207 & $<0.001$ \\
\hline TC & 0.004 & 0.929 & 0.013 & 0.764 & 0.031 & 0.603 & -0.025 & 0.685 & -0.027 & 0.616 & 0.016 & 0.805 \\
\hline TG & -0.035 & 0.382 & -0.032 & 0.454 & -0.148 & 0.306 & -0.077 & 0.188 & -0.005 & 0.933 & -0.000 & 0.999 \\
\hline LDL-C & -0.051 & 0.206 & -0.007 & 0.864 & -0.072 & 0.228 & -0.006 & 0.919 & -0.030 & 0.582 & -0.025 & 0.706 \\
\hline HDL-C & 0.176 & 0.360 & 0.091 & 0.154 & 0.153 & 0.207 & 0.065 & 0.174 & 0.004 & 0.842 & 0.001 & 0.875 \\
\hline P1NP & 0.390 & $<0.001$ & 0.528 & $<0.001$ & 0.305 & $<0.001$ & 0.510 & $<0.001$ & 0.452 & $<0.001$ & 0.506 & $<0.001$ \\
\hline$\beta-C T X$ & 0.405 & $<0.001$ & 0.522 & $<0.001$ & 0.335 & $<0.001$ & 0.543 & $<0.001$ & 0.456 & $<0.001$ & 0.472 & $<0.001$ \\
\hline HOMA-IR & 0.094 & 0.015 & 0.072 & 0.083 & 0.148 & 0.010 & 0.018 & 0.754 & 0.040 & 0.445 & 0.113 & 0.224 \\
\hline HOMA-IS & 0.033 & $<0.001$ & 0.023 & 0.724 & 2.690 & 0.001 & 0.380 & 0.668 & 0.228 & 0.005 & 0.116 & 0.068 \\
\hline HOMA- $\beta$ & 0.130 & 0.035 & 0.140 & 0.002 & 0.170 & 0.025 & 0.082 & 0.144 & 0.014 & 0.785 & 0.157 & 0.011 \\
\hline
\end{tabular}

\section{Discussion}

It is well known that T2DM is a complex metabolic disease caused by the interaction of genetic, environmental and other risk factors. Earlier clinical studies reported serum $\mathrm{OC}$ was associated with glucose tolerance in middle-aged men [5] and T2DM patients [6]. In the present study, serum concentrations of TOC, HbA1C and BMI were statistically different between Han and Uygur T2DM patients. By multivariate analysis, the concentration of TOC was inversely associated with HbA1C for Han and Uygur T2DM participants and it was also inversely correlated with BMI for Uygur T2DM participants. In addition, it was positively associated with HOMA- $\beta$ for Uygur male participants, indicating there is an interaction between TOC and glucose metabolism. The results indicated that genetic factors may play a role in modulating TOC and glucose metabolism in different ethnic population.

Emerging evidences from clinical and animal studies have shown that bone interacts with glucose metabolism by regulating insulin secretion from pancreas as well as adipokines from adipose tissue. As a bone-derived hormone produced and secreted specifically by osteoblasts, OC has been widely used as a biochemical marker of bone formation and bone turnover [11]. Recent studies in mice demonstrated the potential effect of skeleton in glucose and lipid homeostasis, which was regulated by OC,indicating OC may be involved in the regulation of glucose and lipid metabolism in human.

The association between serum OC and energy metabolism has been extensively studied in T2DM patients. However, so far contrasting results have been reported. Iki et al. investigated the association of the serum OC and glycemic status and IR in an elderly Japanese male population and demonstrated that serum OC was negatively correlated with FPG and HbA1C [12]. Kanazawa et al. surveyed 101 postmenopausal women and 152 men diagnosed with T2DM and concluded serum OC was negatively correlated with FPG and HbA1C [13]. However, Wang et al. reported there was no relationship 
Table 4 Multivariable association of TOC with other independent selected variables

\begin{tabular}{|c|c|c|c|c|c|c|}
\hline Groups & Variables & $\beta$ & Sd. E & Standardized $\beta$ & t & $P$ value \\
\hline \multirow[t]{4}{*}{ Total Uygur group } & $\mathrm{HbA1C}$ & -0.663 & 0.191 & -0.180 & -3.468 & 0.001 \\
\hline & P1NP & 0.100 & 0.023 & 0.296 & 4.337 & $<0.001$ \\
\hline & $B-C T X$ & 11.096 & 2.289 & 0.331 & 4.847 & $<0.001$ \\
\hline & BMI & -0.184 & 0.072 & -0.097 & -2.567 & 0.011 \\
\hline \multirow[t]{3}{*}{ Total Han group } & $\mathrm{HbA1C}$ & -0.536 & 0.129 & -0.157 & -4.169 & $<0.001$ \\
\hline & P1NP & 0.097 & 0.015 & 0.313 & 6.363 & $<0.001$ \\
\hline & $\beta-C T X$ & 10.249 & 1.618 & 0.316 & 6.335 & $<0.001$ \\
\hline \multirow[t]{5}{*}{ Uygur Males group } & $\mathrm{HbA1C}$ & -0.691 & 0.202 & -0.247 & -3.979 & 0.002 \\
\hline & $\beta-C T X$ & 0.223 & 0.079 & 0.341 & 2.836 & 0.001 \\
\hline & BMl & -0.454 & 0.167 & -0.171 & -2.893 & 0.014 \\
\hline & FCP & 0.639 & 0.292 & 0.151 & 2.185 & 0.031 \\
\hline & HOMA- $\beta$ & 0.016 & 0.048 & 0.207 & 3.567 & 0.001 \\
\hline \multirow[t]{3}{*}{ Han Males group } & $\mathrm{HbA} 1 \mathrm{C}$ & -0.451 & 0.164 & 0.189 & -2.185 & 0.031 \\
\hline & P1NP & 0.088 & 0.017 & 0.425 & 5.230 & $<0.001$ \\
\hline & $\beta-C T X$ & 6.890 & 1.887 & 0.292 & 3.655 & $<0.001$ \\
\hline \multirow[t]{4}{*}{ Uygur Females group } & $\mathrm{HbA} 1 \mathrm{C}$ & -0.613 & 0.172 & -0.164 & -3.564 & 0.003 \\
\hline & P1NP & 0.070 & 0.028 & 0.278 & 2.499 & 0.015 \\
\hline & $\beta-C T X$ & 11.036 & 3.285 & 0.398 & 3.359 & 0.001 \\
\hline & BMI & -0.320 & 0.142 & -0.158 & -2.250 & 0.027 \\
\hline \multirow[t]{3}{*}{ Han Females group } & $\mathrm{HbA1C}$ & -0.608 & 0.200 & -0.186 & -3.036 & 0.003 \\
\hline & P1NP & 0.071 & 0.024 & 0.240 & 2.994 & 0.003 \\
\hline & $\beta-C T X$ & 13.721 & 2.469 & 0.445 & 4.749 & $<0.001$ \\
\hline
\end{tabular}

between OC and FPG [14]. In our study, TOC and glucose metabolism has been analyzed in 705 Han T2DM patients and 692 Uygur T2DM patients. We found Uygur participants had higher BMI and lower serum TOC than their Han participants. Our result is consistent with Cardiovascular Risk Survey (CRS) study which was performed from June 2007 to March 2010 [15], demonstrating Uygur population had a significantly higher prevalence of obesity and DM than Han population. Life styles, living habits, living environments and genetic factors, especially the genes involved in obesity may contribute to ethnic variance in BMI in the present study. Previous studies have reported obesity contributes to the pathogenesis of T2DM mainly by promoting IR $[16,17]$. The mechanisms whereby obesity predisposes to IR are incompletely understood. Central fat accumulation, intraorgan lipid deposition, plasma free fatty acid accumulation, inflammation and adipocytokine release may contribute to the IR from the adiposity perspective [18-21].

In a previous study conducted in the black, Hispanic and white population enrolled in the Boston Area Community Health/Bone (BACH/Bone) Survey by Travison et al. [22], they found there are racial/ethnic

Table 5 Multivariate stepwise regression analysis of $\mathrm{HbA} 1 \mathrm{C}$ and other parameters

\begin{tabular}{|c|c|c|c|c|c|c|c|c|c|c|c|c|}
\hline \multirow{3}{*}{ Variables } & \multicolumn{6}{|l|}{ Uygur } & \multicolumn{6}{|l|}{ Han } \\
\hline & \multicolumn{2}{|l|}{$\mathrm{HbAC1}$} & \multicolumn{2}{|l|}{ P1NP } & \multicolumn{2}{|l|}{$\beta-C T X$} & \multicolumn{2}{|l|}{$\mathrm{HbA1C}$} & \multicolumn{2}{|l|}{ P1NP } & \multicolumn{2}{|l|}{$\beta-C T X$} \\
\hline & $\bar{\beta}$ & $P$ value & $\beta$ & $P$ value & $\beta$ & $P$ value & $\beta$ & $P$ value & $\bar{\beta}$ & $P$ value & $\beta$ & $P$ value \\
\hline TOC & -0.034 & 0.014 & 0.443 & $<0.001$ & 0.005 & $<0.001$ & -0.045 & 0.015 & 1.933 & $<0.001$ & 0.015 & $<0.001$ \\
\hline FPG & 0.280 & $<0.001$ & - & - & - & - & 0.313 & $<0.001$ & - & - & - & - \\
\hline $2 \mathrm{~h}-\mathrm{PG}$ & 0.098 & $<0.001$ & - & - & - & - & 0.070 & 0.001 & - & - & - & - \\
\hline ALP & - & - & 0.213 & $<0.001$ & 0.002 & $<0.001$ & - & - & 0.190 & 0.001 & 0.002 & $<0.001$ \\
\hline PTH & - & - & 0.152 & 0.003 & 0.001 & 0.041 & - & - & - & - & - & - \\
\hline Calcium & - & - & - & - & -0.206 & 0.033 & - & - & - & - & -0.555 & $<0.001$ \\
\hline
\end{tabular}


Table 6 Multivariate stepwise regression analysis of HOMA- $\beta$ and other parameters among Uygur population

\begin{tabular}{|c|c|c|c|c|c|c|}
\hline \multirow[t]{3}{*}{ Variables } & \multicolumn{4}{|c|}{ Uygur males } & \multirow{2}{*}{\multicolumn{2}{|c|}{$\begin{array}{l}\text { Uygur females } \\
\text { BMI }\end{array}$}} \\
\hline & \multicolumn{2}{|c|}{$\mathrm{BMl}$} & \multicolumn{2}{|c|}{ HOMA- $\beta$} & & \\
\hline & $\beta$ & $P$ value & $\beta$ & $P$ value & $\bar{\beta}$ & $P$ value \\
\hline TOC & -1.563 & 0.011 & 2.101 & 0.040 & -1.284 & 0.016 \\
\hline FPG & - & - & -5.476 & 0.001 & -5.933 & $<0.001$ \\
\hline FCP & 0.906 & 0.016 & - & - & 13.965 & 0.005 \\
\hline FINS & 0.134 & 0.008 & 1.232 & $<0.001$ & - & - \\
\hline
\end{tabular}

heterogeneity in measures of bone mass and density including $\mathrm{OC}$ and concluded that such ethnic heterogeneity may be explained by the variation in body composition, diet and sociodemographic factors. We also found ethnic variation in $\mathrm{OC}$ concentrations between Han and Uygur T2DM patients, which confirmed the results of previous study. Our study demonstrated that TOC was negatively correlated with HbA1C for all Han and Uygur T2DM patients. The inverse association between TOC and HbA1C was retained after adjustment of major risk factors, indicating TOC may modulate glucose metabolism through pathways beyond established risk factors.

$\mathrm{OC}$ has been reported to regulate glucose metabolism by increasing insulin secretion and improving glucose intolerance. Prior animal studies showed that mice lacking OC developed hyperglycemia and glucose intolerance due to IR [2]. The administration of OC in vitro and in wild-type mice could improve glucose homoeostasis by enhancing the expression of insulin genes and genes necessary for $\beta$-cell proliferation $[2,23]$. Kover et al. confirmed OC can protect $\beta$-cells from the negative effects of glucose-induced oxidative stress by reducing TXNIP expression, thereby preserving $\beta$-cell function and survival in rat model [24].

A few studies have investigated the relationship between $\mathrm{OC}$ and glucose metabolism in the general population. In a large cross-sectional analysis of older men aged 70 to 89 years residing in the community of Perth, Western Australia, higher ucOC was found inversely associated with reduced diabetic risk [25]. Shu et al. found $\mathrm{OC}$ at baseline was negatively related to the risk of incident T2DM during a 3-year follow-up [26]. In addition, maternal oral administration of ucOC during gestation protected high-fat, high-sucrose diet-fed female offspring from metabolic disorders induced by maternal obesity [27]. All these studies support a role for OC as a modulator of glucose metabolism, indicating the mechanistic link between $\mathrm{OC}$ and glucose metabolism.

The association between serum OC and BMI has been comprehensive studied in obesity animal model [28]. Only some clinical studies investigated the association of OC and BMI $[6,29,30]$. Hu et al. investigated the association of serum TOC and plasma glucose and BMI in a total of 2032 healthy Chinese women in Shanghai [31], and demonstrated that serum TOC was negatively correlated with BMI. Chin KY et al. surveyed serum TOC and indices of obesity and lipid parameters in a total of 373 aging men from the Malaysian, and indicated that low serum TOC level was more likely to be associated with high BMI [32]. To the best of our knowledge, it was the first study to examine the association between TOC and BMI in Chinese Uygur population. In the present study, we showed Uygur T2DM patients had higher BMI than their Han counterparts, and TOC was negatively associated with BMI for all Uygur T2DM patients, which was consistent with previous studies. However, such relationship has been found only in Han female participants. A possible reason for this discrepancy between ethnicity may be related to the higher prevalence of obesity and unhealthy life styles that were specific to Uygur population. Until now, the correlation between serum $\mathrm{OC}$ and BMI is not completely understood. Further studies are needed to confirm the relationship between $\mathrm{OC}$ and $\mathrm{BMI}$.

Prior animal studies showed that $\mathrm{OC}$ induces insulin release in $\beta$-cells, improves insulin sensitivity and directly regulates metabolic phenotype $[2,23]$. OC knockout mice showed a profoundly deranged metabolic phenotype including IR, glucose intolerance and abnormal increased visceral fat [23]. While given OC, models of obese mice gained significantly less weight and smaller fat pads [23]. Ma et al. reported the serum OC was positively correlated with HOMA- $\beta$ in 495 T2DM males [33]. Kunutsor et al. conducted a systematic review and proved serum OC level was positively associated with HOMA- $\beta$ [34]. Our results confirmed these studies. In the present study, we showed that TOC was positively correlated with HOMA- $\beta$ for Uygur male participants. Our result supported the hypothesis that OC could enhance $\beta$-cells function. Such relationship has not been found in Han and Uygur female participants, which may be related to the limited number of female T2DM patients and the hypothesis that serum oestrogen may disturb the metabolism of OC. In Han male participants, we found no relationship between TOC and HOMA- $\beta$. Different ethnic origin and genetic background may be helpful to explain such ethnic variance.

Dyslipidemia, commonly present in patients with DM, is a well-known atherosclerotic cardiovascular disease (ASCVD) risk factors. For patients with ASCVD or with a moderately increased 10-year risk of ASCVD events, aggressive LDL-lowering is the main pharmacological treatment model. However, some patients with CAD still failed to achieve the target level of lipid levels despite taking aggressive LDL-lowering medicine. Nowadays, nutraceuticals and functional food ingredients have been recommended as adjuvants in reducing overall 
cardiovascular risk induced by dyslipidemia [35]. OC have been reported to play an important role in energy metabolism. While it remained controversial as to whether there was significant association between serum OC and lipid metabolism. Zhou et al. surveyed the association of serum OC and lipid parameters in Chinese subjects, and concluded that HDL-C and TG level were inversely associated with serum OC in Chinese males [36]. Kanazawa et al. reported serum $\mathrm{OC}$ was negatively associated with triglyceride in a longitudinal study conducted in Japan T2DM patients [37]. While Bao et al. found no relationship between serum OC levels and lipid metabolism in subjects with normal glucose tolerance and normal BMI in Chinese men [30]. Brownbill et al. also found no relationship between OC level and serum TG [38]. Our study also failed to find the association between serum $\mathrm{OC}$ and lipid profiles in Chinese Han and Uygur T2DM patients, which may be due to the difference in diet specific to ethnic population and the selection standard or criteria for the subjects in different studies.

More than 7 chromosomal loci associated with T2DM have been identified to date in the populations of European by genome-wide association (GWA) studies [39-43]. Considering the distinct ethnic origin and genetic background between Han and Uygur population, and the important role played by $\mathrm{OC}$ in regulating glucose metabolism, further studies are needed to study the role of polymorphism of $\mathrm{OC}$ genes in modulating susceptibility to DM in Chinese Han and Uygur population.

This study should be viewed in light of several limitations. First, the lack of a healthy control group is the main limitation of our study. Second, this study was a single-center experience and enrolled only Chinese Han and Uygur population. Whether our results can be applied to other races remains to be determined. Third, we were unable to determine the impact of lifestyle on the association between $\mathrm{OC}$ and metabolic parameters. Finally, we did not measure ucOC and assess the direct effect of ucOC on glucose metabolism.

\section{Conclusion}

In summary, our study demonstrated that there was significantly different association between TOC and glucose metabolism and $\beta$-cells function in Han and Uygur T2DM patients, indicating genetic factors may play a role in modulating TOC and glucose metabolism. Further studies are needed to explore the impact of TOC on glucose metabolism in different ethnic humans.

\section{Abbreviations}

25(OH)VD3: 25-hydroxyvitamin D3; 2 h-PG: 2 h-post OGTT plasma glucose; ALP: Alkaline phosphatase; BMD: Bone mass density; BMI: Body mass index; DBP: Diastolic blood pressure; FCP: Fasting plasma C-peptide; FINS: Fasting plasma insulin; FPG: Fasting plasma glucose; HbA1C: Glycated hemoglobin; HDL-C: High density lipoprotein-cholesterol; HOMA-IR: Homeostasis model assessment of insulin resistance; HOMA-IS: Homeostasis model assessment of insulin sensitivity; HOMA- $\beta$ : Homeostasis model assessment of $\beta$-cell function; LDL-C: Low density lipoprotein-cholesterol; P1NP: Procollagen type I Nterminal propeptide; PTH: Parathyroid hormone; SBP: Systolic blood pressure; SD: Standard deviation; T2DM: Type 2 diabetes mellitus; TC: Total cholesterol; TG: Triglyceride; TOC: Serum total osteocalcin; WC: Waist circumference; $\beta$ CTX: $\beta$-C-terminal telopeptide of type I collagen

\section{Acknowledgments}

We gratefully acknowledge Professor Xinling Wang, the director of the Department of Endocrinology, People's Hospital of Xinjiang Uygur Autonomous Region, for her extremely constructive advice on the present study.

\section{Funding}

Not applicable.

\section{Availability of data and materials}

The datasets generated during the present study are not publicly available, because detailed clinical information of each participant were included in these materials.

\section{Authors' contributions}

YX conceived the study design, and completed the quality of data. $Y C$ and QZ performed the experiments, collected the data, and wrote the paper. GD performed the statistical analysis. All authors read and approved the final manuscript.

\section{Competing interests}

The authors declared that there was no conflict of interests in this study.

\section{Consent for publication}

Not applicable.

\section{Ethics approval and consent to participate}

The Ethics Review Committee of People's Hospital of Xinjiang Uygur Autonomous Region approved this study (Urumqi, China; 6 December 2013), and all procedures were conducted in accordance with the Declaration of Helsinki. All subjects were fully informed of the study aims and design and a written informed consent was obtained from each participant.

\section{Author details}

'Department of Endocrinology, Zhongnan Hospital of Wuhan University, 169 Donghu Road, Wuhan, Hubei 430071, China. ${ }^{2}$ Department of Endocrinology, People's Hospital of Xinjiang Uygur Autonomous Region, Urumqi, China. ${ }^{3}$ Department of Cardiology, First Affiliated Hospital of Xinjiang Medical University, Urumqi, China. ${ }^{4}$ Xinjiang Key Laboratory of Cardiovascular Disease Research, Urumqi, China. ${ }^{5}$ Department of Endocrinology, First Affiliated Hospital of Xinjiang Medical University, Urumqi, China.

Received: 23 November 2016 Accepted: 5 June 2017

Published online: 21 July 2017

References

1. Nyman JS, Even JL, Jo CH, Herbert EG, Murry MR, Cockrell GE, et al. Increasing duration of type 1 diabetes perturbs the strength-structure relationship and increases brittleness of bone. Bone. 2011:48:733-40.

2. Lee NK, Sowa H, Hinoi E, Ferron M, Ahn JD, Confavreux C, et al. Endocrine regulation of energy metabolism by the skeleton. Cell. 2007:130:456-69.

3. Ducy P, Amling M, Takeda S, Priemel M, Schilling AF, Beil FT, et al. Leptin inhibits bone formation through a hypothalamic relay: a central control of bone mass. Cell. 2000;100:197-207.

4. Elefteriou F, Ahn JD, Takeda S, Starbuck M, Yang X, Liu X, et al. Leptin regulation of bone resorption by the sympathetic nervous system and CART. Nature. 2005:434:514-20.

5. Hwang YC, Jeong IK, Ahn KJ, Chung HY. The uncarboxylated form of osteocalcin is associated with improved glucose tolerance and enhanced beta-cell function in middle-aged male subjects. Diabetes Metab Res Rev. 2009;25:768-72.

6. Kanazawa I, Yamaguchi T, Yamauchi M, Yamamoto M, Kurioka S, Yano S, et al. Serum undercarboxylated osteocalcin was inversely associated with plasma glucose level and fat mass in type 2 diabetes mellitus. Osteoporos Int. 2011;22:187-94. 
7. Liatis S, Sfikakis PP, Tsiakou A, Stathi C, Terpos E, Katsilambros N, et al. Baseline osteocalcin levels and incident diabetes in a 3-year prospective study of high-risk individuals. Diabetes Metab. 2014;40:198-203.

8. Thrailkill KM, Jo CH, Cockrell GE, Moreau CS, Lumpkin CK Jr, Fowlkes JL. Determinants of undercarboxylated and carboxylated osteocalcin concentrations in type 1 diabetes. Osteoporos Int. 2012;23:1799-806.

9. Wang X, Chen J, Li L, Zhu CL, Gao J, Rampersad S, et al. New association of bone morphogenetic protein 4 concentrations with fat distribution in obesity and Exenatide intervention on it. Lipids Health Dis. 2017;16(1):70.

10. Matthews DR, Hosker JP, Rudenski AS, Naylor BA, Treacher DF, Turner RC. Homeostasis model assessment: insulin resistance and beta-cell function from fasting plasma glucose and insulin concentrations in man. Diabetologia. 1985;28:412-9.

11. Avbersek-Luznik I, Gmeiner Stopar T, Marc J. Activity or mass concentration of bone-specific alkaline phosphatase as a marker of bone formation. Clin Chem Lab Med. 2007:45:1014-8.

12. Iki M, Tamaki J, Fujita Y, Kouda K, Yura A, Kadowaki E, et al. Serum undercarboxylated osteocalcin levels are inversely associated with glycemic status and insulin resistance in an elderly Japanese male population: Fujiwara-kyo osteoporosis risk in men (FORMEN) study. Osteoporos Int. 2012;23:761-70.

13. Kanazawa I, Yamaguchi T, Tada Y, Yamauchi M, Yano S, Sugimoto T. Serum osteocalcin level is positively associated with insulin sensitivity and secretion in patients with type 2 diabetes. Bone. 2011;48:720-5.

14. Wang $Q$, Zhang $B, X u Y, X u$ H, Zhang $N$. The relationship between serum osteocalcin concentration and glucose metabolism in patients with type 2 diabetes mellitus. Int J Endocrinol. 2013;2013:842598.

15. Lai HM, Li XM, Yang YN, Ma YT, Xu R, Pan S, et al. Genetic variation in NFKB1 and NFKBIA and susceptibility to coronary artery disease in a Chinese Uygur population. PLoS One. 2015;10:e0129144.

16. Alexander CM, Landsman PB, Grundy SM. Metabolic syndrome and hyperglycemia: congruence and divergence. Am J Cardiol. 2006;98:982-5.

17. Reaven GM. Banting lecture 1988. Role of insulin resistance in human disease. Diabetes. 1988;37:1595-607.

18. Ouchi N, Parker JL, Lugus JJ, Walsh K. Adipokines in inflammation and metabolic disease. Nat Rev Immunol. 2011:11:85-97.

19. Bremer AA, Jialal I. Adipose tissue dysfunction in nascent metabolic syndrome. J Obes. 2013;2013:393192.

20. InterAct C, Langenberg C, Sharp SJ, Schulze MB, Rolandsson O, Overvad K, et al. Long-term risk of incident type 2 diabetes and measures of overall and regional obesity: the EPIC-InterAct case-cohort study. PLoS Med. 2012;9:e1001230.

21. Zhang $\mathrm{H}$, Zhang C. Adipose "talks" to distant organs to regulate insulin sensitivity and vascular function. Obesity (Silver Spring). 2010;18:2071-6.

22. Travison TG, Chiu GR, McKinlay JB, Araujo AB. Accounting for racial/ethnic variation in bone mineral content and density: the competing influences of socioeconomic factors, body composition, health and lifestyle, and circulating androgens and estrogens. Osteoporos Int. 2011;22:2645-54.

23. Ferron M, Hinoi E, Karsenty G, Ducy P. Osteocalcin differentially regulates beta cell and adipocyte gene expression and affects the development of metabolic diseases in wild-type mice. Proc Natl Acad Sci U S A. 2008;105:5266-70.

24. Kover K, Yan Y, Tong PY, Watkins D, Li X, Tasch J, et al. Osteocalcin protects pancreatic beta cell function and survival under high glucose conditions. Biochem Biophys Res Commun. 2015;462:21-6.

25. Yeap BB, Alfonso H, Chubb SA, Gauci R, Byrnes E, Beilby JP, et al. Higher serum undercarboxylated osteocalcin and other bone turnover markers are associated with reduced diabetes risk and lower estradiol concentrations in older men. J Clin Endocrinol Metab. 2015;100:63-71.

26. Shu H, Pei Y, Chen K, Lu J. Significant inverse association between serum osteocalcin and incident type 2 diabetes in a middle-aged cohort. Diabetes Metab Res Rev. 2016;32:867-74.

27. Kawakubo-Yasukochi T, Kondo A, Mizokami A, Hayashi Y, Chishaki S, Nakamura S, et al. Maternal oral administration of osteocalcin protects offspring from metabolic impairment in adulthood. Obesity (Silver Spring). 2016;24:895-907

28. Kim YS, Nam JS, Yeo DW, Kim KR, Suh SH, Ahn CW. The effects of aerobic exercise training on serum osteocalcin, adipocytokines and insulin resistance on obese young males. Clin Endocrinol. 2015;82:686-94.

29. Saleem U, Mosley TH Jr, Kullo IJ. Serum osteocalcin is associated with measures of insulin resistance, adipokine levels, and the presence of metabolic syndrome. Arterioscler Thromb Vasc Biol. 2010;30:1474-8.
30. Bao Y, Ma X, Yang R, Wang F, Hao Y, Dou J, et al. Inverse relationship between serum osteocalcin levels and visceral fat area in Chinese men. J Clin Endocrinol Metab. 2013;98:345-51.

31. Hu WW, Ke YH, He JW, Fu WZ, Liu YJ, Chen D, et al. Serum osteocalcin levels are inversely associated with plasma glucose and body mass index in healthy Chinese women. Acta Pharmacol Sin. 2014;35:1521-6.

32. Chin KY, Ima-Nirwana S, Mohamed IN, Ahmad F, Ramli ES, Aminuddin A et al. Serum osteocalcin is significantly related to indices of obesity and lipid profile in Malaysian men. Int J Med Sci. 2014;11:151-7.

33. Ma XY, Chen FQ, Hong H, LV XJ, Dong M, Wang QY. The relationship between serum osteocalcin concentration and glucose and lipid metabolism in patients with type 2 diabetes mellitus - the role of osteocalcin in energy metabolism. Ann Nutr Metab. 2015;66:110-6.

34. Kunutsor SK, Apekey TA, Laukkanen JA. Association of serum total osteocalcin with type 2 diabetes and intermediate metabolic phenotypes: systematic review and meta-analysis of observational evidence. Eur J Epidemiol. 2015;30:599-614.

35. Scicchitano P, Cameli M, Maiello M, Modesti PA, Muiesan ML, Novo S, et al. Nutraceuticals and dyslipidaemia: beyond the common therapeutics. J Funct Foods. 2014;6:11-32.

36. Zhou M, Ma X, Li H, Pan X, Tang J, Gao Y, et al. Serum osteocalcin concentrations in relation to glucose and lipid metabolism in Chinese individuals. Eur J Endocrinol. 2009;161:723-9.

37. Kanazawa I, Yamaguchi T, Sugimoto T. Relationship between bone biochemical markers versus glucose/lipid metabolism and atherosclerosis; a longitudinal study in type 2 diabetes mellitus. Diabetes Res Clin Pract. 2011;92:393-9.

38. Brownbill RA, llich JZ. Lipid profile and bone paradox: higher serum lipids are associated with higher bone mineral density in postmenopausal women. J Womens Health (Larchmt). 2006; 15:261-70.

39. Wellcome Trust Case Control C. Genome-wide association study of 14,000 cases of seven common diseases and 3,000 shared controls. Nature. 2007:447:661-78

40. Diabetes Genetics Initiative of Broad Institute of H, Mit LU, Novartis Institutes of BioMedical R, Saxena R, Voight BF, Lyssenko V, Burtt NP, de Bakker PI, Chen H, Roix JJ, et al. Genome-wide association analysis identifies loci for type 2 diabetes and triglyceride levels. Science. 2007; 316:1331-6.

41. Scott LJ, Mohlke KL, Bonnycastle LL, Willer CJ, Li Y, Duren WL, et al. A genome-wide association study of type 2 diabetes in Finns detects multiple susceptibility variants. Science. 2007;316:1341-5

42. Sladek R, Rocheleau G, Rung J, Dina C, Shen L, Serre D, et al. A genomewide association study identifies novel risk loci for type 2 diabetes. Nature. 2007:445:881-5

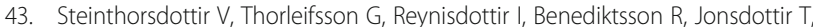
Walters $G B$, et al. A variant in CDKAL1 influences insulin response and risk of type 2 diabetes. Nat Genet. 2007;39:770-5.

\section{Submit your next manuscript to BioMed Central and we will help you at every step:}

- We accept pre-submission inquiries

- Our selector tool helps you to find the most relevant journal

- We provide round the clock customer support

- Convenient online submission

- Thorough peer review

- Inclusion in PubMed and all major indexing services

- Maximum visibility for your research

Submit your manuscript at www.biomedcentral.com/submit 